\title{
El espacio de articulación urbana-rural de Buenos Aires en el siglo XIX
}

\begin{tabular}{|c|c|c|}
\hline \multicolumn{2}{|c|}{ Fecha de recepción: 13 de octubre de 2016 Fecha de aceptación: 31 de julio de 2017} & Disponible en línea: 5 de febrero de 2018 \\
\hline \multicolumn{2}{|l|}{ Patricia López Goyburu } & $\begin{array}{l}\text { Consejo Nacional de Investigaciones } \\
\text { Científicas y Tecnológicas (Conicet) }\end{array}$ \\
\hline $\begin{array}{l}\text { Instituto de Arte Americano } \\
\text { (FADU-UBA) }\end{array}$ & $\begin{array}{l}\text { ORCID: https://orcid.org/0000- } \\
\text { 0002-2120-2758 }\end{array}$ & plopezgoyburu@gmail.com \\
\hline
\end{tabular}

Resumen A lo largo de la historia de Buenos Aires se observan diferentes tipos de articulación entre el sistema urbano y el rural. En el presente trabajo se expone cómo se ve y se proyecta la relación entre el sistema urbano y el rural en esa ciudad durante el siglo XIX. Para ello, es necesario remitirse al estudio de los documentos urbanísticos y bibliográficos que abordan esta época. El estudio muestra que la cartográfica fue el instrumento utilizado para geometrizar el territorio, lo que facilitó el control técnico y administrativo.

Palabras clave: articulación urbano-rural; planificación; expansión urbana; Buenos Aires

\section{The Urban-Rural Articulation}

Space of Buenos Aires in the Nineteenth Century

Abstract Throughout the history of Buenos Aires there are different types of articulation between the urban and the rural system. The present work aims to understand how the relationship between the urban and the rural system is looked and projected in Buenos Aires in the nineteenth century. For that reason it is necessary to refer to the study of urban and bibliographic documents dealing with the city in this period. The study shows that the mapping was the instrument used to geometrize the territory and this facilitated the technical and administrative control.

Keywords urban-rural articulation; planning; urban sprawl; Buenos Aires 


\section{0 espaço de articulação}

urbano-rural de Buenos Aires no século XIX

Resumo Ao longo da história de Buenos Aires são observados diferentes tipos de articulação entre o sistema urbano e o rural. Este artigo tem como objetivo compreender como a relaçáo entre sistemas urbanos e rurais em Buenos Aires no século 19 é vista e projetada. Para isso, é necessário consultar o estudo dos documentos urbanísticos e bibliográficos que se aproximam desta época da cidade. $\mathrm{O}$ estudo mostrou que a cartografia era o instrumento usado para geometrizar o território e isso facilitava o controle técnico e administrativo.

Palavras chaves articulação urbana rural; planejamento; expansão urbana; Buenos Aires 


\section{Introducción}

En 1820, Buenos Aires ocupaba una superficie de $3 \mathrm{~km}$ de largo y $2 \mathrm{~km}$ de ancho. La ciudad contaba con 50.000 habitantes que se aglomeraban en torno a la plaza de Mayo. En esta época, la ciudad se encontraba en el punto de inflexión entre la aldea y la metrópolis; entre un espacio colonial ordenado y un espacio metropolitano desordenado en donde el mercado urbano, político y cultural se transformaba y complejizaba. Esta tensión en la que se encontraba la ciudad frente a la expansión urbana centra la mirada en el espacio de articulación entre el sistema urbano y el sistema rural.

El artículo propone una revisión del espacio de articulación urbano-rural de Buenos Aires en el siglo XIX a partir de documentos urbanísticos y bibliográficos.

El presente trabajo, que es tributario de investigaciones precedentes, tiene los siguientes objetivos:

, Revisar los documentos urbanísticos y bibliográficos que abordan la ciudad de Buenos Aires durante el siglo XIX.

, Analizar y evaluar dicha información.

, Caracterizar y procesar la información significativa relacionada con el espacio de articulación urbano-rural.La investigación es de tipo cualitativo, en la medida en que lo que se persigue es explicar las estrategias de intervención en la articulación entre el sistema urbano y rural de Buenos Aires en el siglo XIX. El estudio se hará por medio de cartografía y documentos urbanísticos y de un análisis de las interacciones entre las condiciones rurales y urbanas en el período seleccionado.

El método de investigación se compone de dos fases: en la primera se buscan los documentos bibliográficos y urbanísticos que comprenden el lapso de estudio y, en la segunda, se analizan y evalúan los hallazgos.

Los planes y planos manifiestan las formas de pensar la ciudad en cada momento histórico. Estos actúan como mediadores para indagar acerca de cómo se piensa la relación ciudad-no ciudad y su revisión crítica permite descubrir las "leyes" de articulación entre el espacio urbano y el espacio rural que marcan esta etapa.

\section{El crecimiento de la ciudad en el siglo XIX}

Buenos Aires recoge las aguas de tres importantes cuencas que vienen del oeste (ríos Luján, Reconquista y Matanza-Riachuelo); además, su red hidrográfica está constituida por un gran número de arroyos que crean bañados y tierras inundables, por lo que la hidrografía y la topografía estructuran el territorio.

A principios del siglo XIX, el crecimiento seguía los condicionantes del medio físico. La ciudad se extendía atendiendo las partes más elevadas y las barrancas continuaban limitando su desarrollo hacia el norte y hacia el sur; por eso, se desplegaba hacia el oeste sobre la horizontalidad de la 
llanura (Sociedad Central de Arquitectos, 1955) (Figura 1).

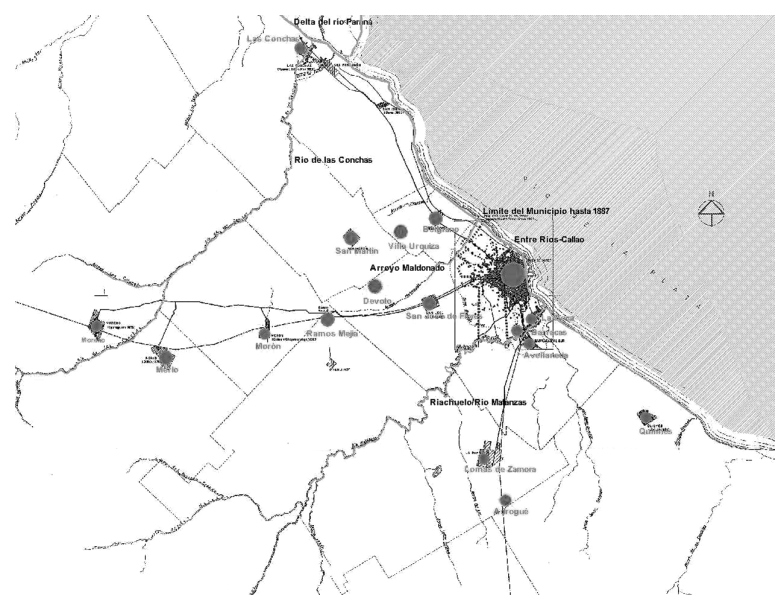

Figura 1. Plano de localización de barrios de la ciudad de Buenos Aires y ciudades de área metropolitana sobre plano de César Vapñarsky, 1870.

Fuente: Vapñarsky (2000)

El soporte físico condicionaba el desarrollo de los núcleos urbanos. Las fábricas de productos alimenticios y manufacturas de sebo, suelas, curtidurías, jabón, fósforos y papel, entre otros, propiciaban el crecimiento de Avellaneda y Quilmes; las industrias de la leche y afines, el de los pueblos de Merlo y Moreno y las casaquintas, en Adrogué, Ramos Mejía, Morón y San Martín.

En 1821, Rivadavia creó un departamento de ingenieros-arquitectos para hacerse cargo de la planificación de la obra pública. La zona sur poseía tierras anegadizas que requirieron grandes obras hidráulicas y de relleno para ser saneadas y urbanizadas. Aliata (2006) muestra que las aspiraciones de comunicación universal, de mejora industrial y de dominio del ser humano sobre la naturaleza aparecían como aspectos de fe en el progreso que movieron las intervenciones sobre el territorio hasta fines del siglo.

La ciudad se expandía fundamentalmente hacia el oeste y hacia el norte. Esta expansión urbana centró mirada en el espacio de articulación entre el sistema urbano y el sistema rural. Holl
(1991) Batty y Longley (1994) y Balcells y Bru (2002) sostienen que el espacio de articulación urbano-rural resuelve y materializa los límites espaciales y funcionales en el territorio y refleja la capacidad de organización de los terrenos que él vincula o delimita. Estos territorios son lugares topológicamente problemáticos.

Tal espacio de articulación es un escenario de interacción entre flujos rurales y urbanos; son lugares donde los nexos urbano-rurales, los cambios y los conflictos económicos, sociales y ambientales son más intensos. En esta línea, Eizaguirre (2001) plantea que el territorio está constituido por la integración del sistema urbano y rural, mientras el espacio de articulación urbano-rurales la zona de contacto entre ambos sistemas, en el cual las estructuras rurales se subruralizan $y$ las estructuras urbanas se suburbanizan.

La concepción de este medio como articulador entre ambos sistemas da lugar a un nuevo tipo de marco que, al igual que el urbano y el rural, también se modela en el tiempo y en el espacio (Allen, 2003; Toro, Velasco y Niño, 2005) (Figuras 2 y 3). A lo largo de la historia del espacio de articulación urbano-rural de Buenos Aires se aplican diferentes tipos de límites entre el sistema urbano y el rural, cuyos alcances varían entre momentos históricos (López, 2016; 2017).

\section{El espacio de articulación entre el sistema urbano y el sistema rural}

A fines del siglo XVIII, los cambios en la técnica de gestión y proyecto provocaron modificaciones en la forma de operar en la ciudad. Aliata (2006) afirma que las técnicas urbanas buscaron determinar el crecimiento de la ciudad y sus límites, a fin de garantizar el funcionamiento de la estructura productiva. 


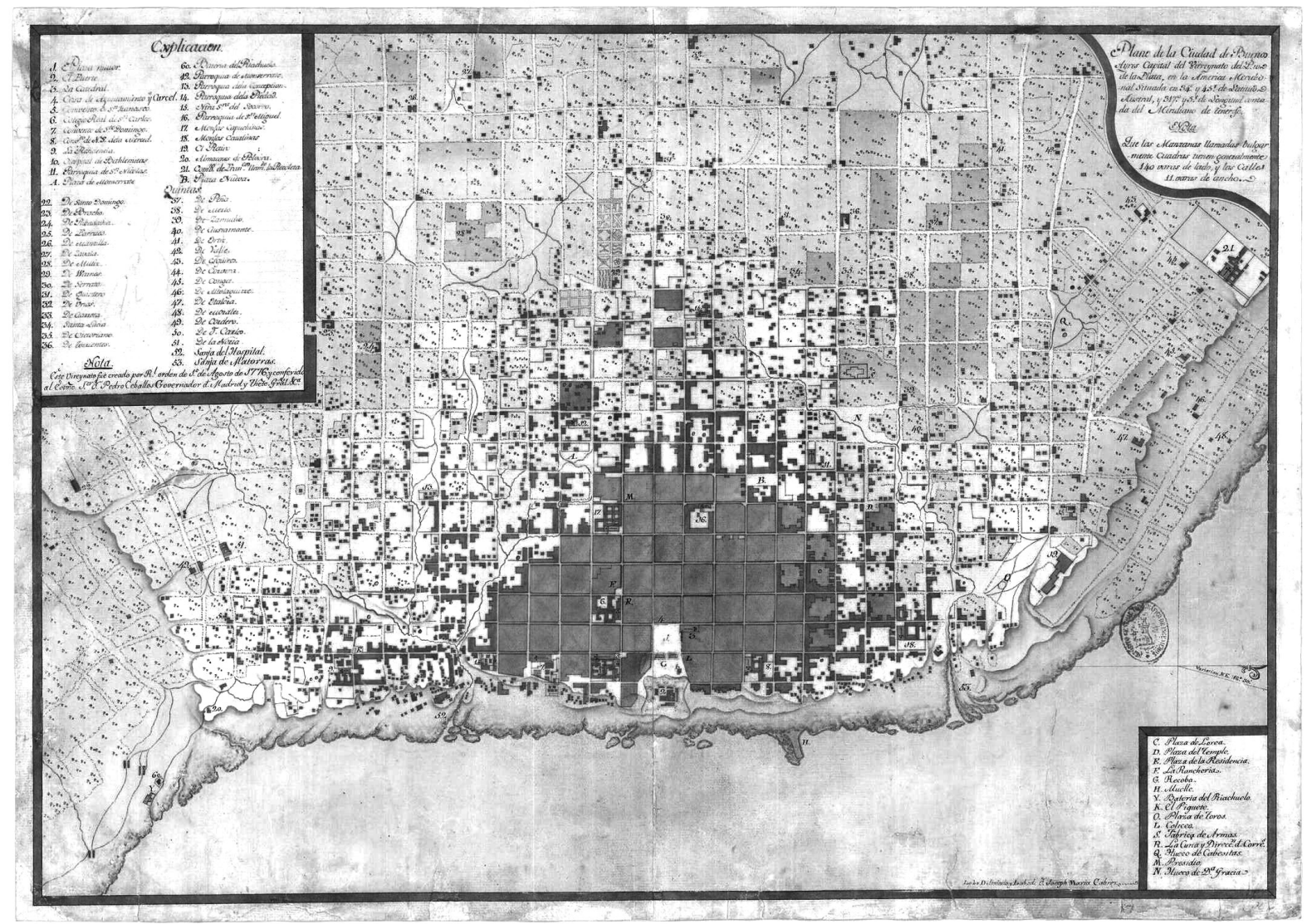

Figura 2. Plano de la ciudad de Buenos Aires (1776).

Fuente: Plano de la ciudad de Buenos Aires de Josep María Cobrel J. (1776). [Imagen en archivo]. Fichero del Servicio Histórico Militar de Madrid, con referencia ARG-07/04.

Este pensamiento sobre una organización urbana racional y simple se acrecentó en 1750 por el descubrimiento de los peligros higiénicos. La emergencia de epidemias en Buenos Aires, sumada a la corriente higienista que circulaba por Europa, hizo tomar conciencia sobre las condiciones de vida urbana; por ello, se hizo especial énfasis en la importancia de mejorar las condiciones de higiene de la ciudad, de establecer una red de distribución de agua potable y de construir una red cloacal.

La necesidad de consolidar el vasto territorio implicó un cambio en el concepto de regularidad que llevó a una transformación física de la ciudad. Tal concepto, como sinónimo de orden, estaba presente en los documentos de la época. Se consideraba que la cuadrícula y las formas geométricas perfectas iban a provocar la 'regularidad de la población'. En ese contexto, la primera acción que produjo el Gobierno provincial fue la elaboración de un plano topográfico.

Las ordenanzas reales de 1786 ensancharon el espacio de articulación, al desplazar los cementerios fuera de la ciudad y, paulatinamente, los hospitales.

Rivadavia ${ }^{1}$ estableció una política de gestión organizadora de la ciudad, con el propósito de introducir costumbres civilizadoras. En 1824 se sancionó la Ley de Enfiteusis ${ }^{2}$, que impulsó la

Primer presidente de la Nación Argentina (8 de febrero de 1826-7 de julio de 1827).

2 Ley dictada por Bernardino Rivadavia (1822-1826) por la cual el Estado conserva la propiedad de las tierras públicas y entrega al enfiteuta una determinada extensión para ser trabajada por no menos de veinte años (aunque no a perpetuidad) mediante el pago de un canon (alquiler anual). 


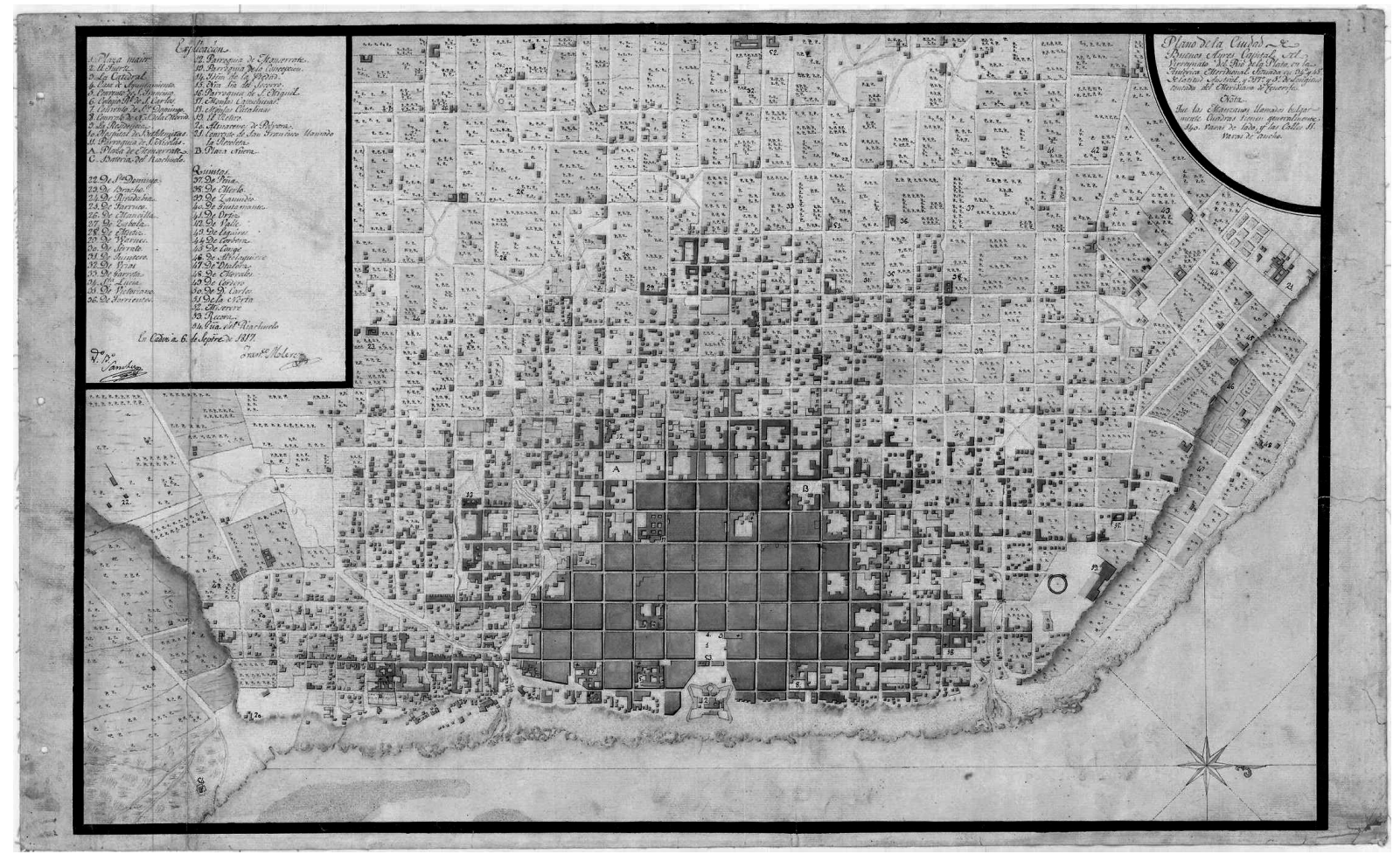

Figura 3. Plano de la ciudad de Buenos Aires (1817).

Fuente: Plano de la ciudad de Buenos Aires, anónimo. (1817). [Imagen en archivo]. Fichero del Servicio Histórico Militar de Madrid, con referencia ARG-07/01.

organización topográfica del territorio con bases científicas y estableció los temas relacionados con el desarrollo físico de la ciudad. Más adelante, en 1827, instituyó el Boulevard de circunvalación para delimitar el espacio posible de racionalización y control.

En los planos de regularización se reconocen dos estrategias principales que actúan sobre el espacio de articulación urbano-rural: la retícula, que genera una trama flexible para regular los suburbios y los planos topográficos, para adaptarse a la latencia de este escenario. En un contexto de crecimiento desordenado sobre el territorio, la falta de un registro que permitiera conocer qué terrenos estaban ocupados dificultaba el accionar de la Administración pública.

Frente a la incapacidad de la cuadrícula de responder a la expansión urbana caracterizada por la irregularidad espacial, se buscó una resemantización de la cuadrícula renacentista traída por las leyes de Indias. Se introdujeron mecanismos que permitieron prescindir de la completa rectitud de la trama urbana, así como de la distribución jerárquica de espacios. La cuadrícula se convirtió en una trama abierta que podía expandirse y repetirse sobre un espacio más vasto.

En cuanto a los planos topográficos, en 1826, José María Romero (arquitecto ingeniero de la ciudad) comenzó a elaborar el plano de la "parte exterior", con el fin de restaurar el trazado "regular". Se disolvió la estructura que fijaba la distribución de usos en la época colonial y regulaba el borde. Estos planos tenían como principal finalidad controlar el desarrollo físico de la ciudad (Figura 4). 


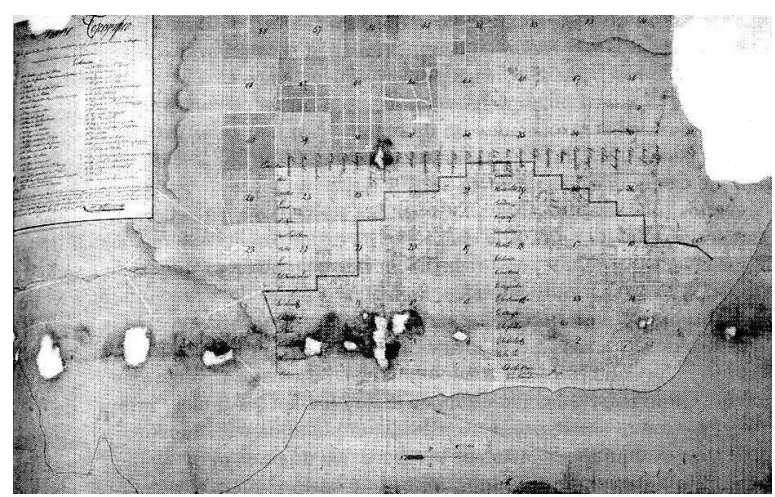

Figura 4. Plano de José María Romero. 1824. ASRE, AZ, 151. Fuente: Aliata (2006)

\section{Consideraciones finales}

En esta época, la cartografía fue el instrumento utilizado para geometrizar el territorio y esto facilitó el control técnico y administrativo del espacio de articulación urbano-rural. La extensión de la retícula intensificó la disolución de los términos estructurantes del 'ejido' de la ciudad colonial.

La cuadrícula perfecta y la precisa distribución de usos de la ciudad colonial van dejando lugar a una trama abierta que, apoyada en la alineación de las calles y la normativa, permitía adaptarse a la expansión de la ciudad.

Esta nueva geometría llevó a ordenar y despojar de los valores simbólicos de la época colonial. El orden regular establecido por las leyes de Indias fue reinterpretado por los técnicos iluministas (ingenieros, arquitectos, urbanistas, geógrafos, topógrafos e higienistas). En los primeros años de la década del veinte, la expansión de la ciudad continuó trasladando el tema suburbano fuera de los límites jurídicos y administrativos de la ciudad. La retícula permitió generar una trama flexible que posibilitó regularlos suburbios y los planos topográficos posibilitaron crear herramientas flexibles.

\section{Bibliografía}

Aliata, F. (2006). La ciudad regular. Arquitectura, programas e instituciones en el Buenos Aires posrevolucionario, 1821-1835. Buenos Aires: Prometeo.

Allen, A. (2003).Environmental Planning and Management of the Peri-Urban Interface: Perspectives on an Emerging Field. Environment and Urbanization, 15(1), 135-147.

Balcells, C. y Bru, J. (2002). Al lado de. Limites, bordes y fronteras. Barcelona:Gustavo Gili.

Batty, M. y Longley, P. (1994). Fractal Cities. A Geometry of Form and Function. Londres: Academic Press Limited.

Eizaguirre, X. (2001). La construcción del territorio disperso. Talleres de reflexión sobre la forma difusa. Barcelona: Edicions UPC.

Holl, S. (1991). Edge of a City. Nueva York: Princeton Architectural Press.

López, P. (2016). El periurbano de Buenos Aires hacia 1925. El caso del Plan de la Comisión de Estética Edilicia. Párrafos Geográficos, 15(2), 73-85. Recuperado de http://www.igeopat.org/ parrafosgeograficos/index.php?option $=\mathrm{com}_{-}$ content $\&$ view $=$ article $\& \mathrm{id}=56$ : articulos $-26 \& \mathrm{ca}-$ tid=26:pg2016-15-2

López, P. (2017). El espacio de articulación urbano-rural de Buenos Aires a mediados del siglo XX y el Plan Regulador de 1958. Estudios Socioterritoriales, 21. Recuperado de http://www. scielo.org.ar/scielo.php?script=sci_arttext\&pi$\mathrm{d}=$ S1853-43922017000100008\&lng=es\&tln$\mathrm{g}=\mathrm{es}$

Sociedad Central de Arquitectos (Buenos Aires). (1955). Evolución del Gran Buenos Aires en el 
tiempo y en el espacio. Revista de Arquitectura, (375), 1-88.

Toro, C., Velasco, V. y Niño, A. (2005). El borde como espacio articulador de la ciudad actual y su entorno. Revista ingenierias Universidad de Medellin, 4(7), 55-65. Recuperado de http://www. redalyc.org/html/750/75004705/
Vapñarsky, C. A. (2000). La aglomeración Gran Buenos Aires. Expansión espacial y crecimiento demográfico entre 1869 y 1991. Buenos Aires: Eudeba. 